A N N A L E S Annales de Bretagne et des Pays de l'Ouest

\title{
Jean-Pierre LethuILLIER (dir), Des habits et nous. Vêtir nos identités
}

\section{Brigitte Maillard}

\section{OpenEdition}

\section{Journals}

Édition électronique

URL : https://journals.openedition.org/abpo/299

DOI : 10.4000/abpo.299

ISBN : 978-2-7535-1511-6

ISSN : 2108-6443

Éditeur

Presses universitaires de Rennes

Édition imprimée

Date de publication : 30 septembre 2008

Pagination : 201-202

ISBN : 978-2-7535-0772-2

ISSN : 0399-0826

Référence électronique

Brigitte Maillard, « Jean-Pierre Lethuiluter (dir), Des habits et nous. Vêtir nos identités », Annales de Bretagne et des Pays de l'Ouest [En ligne], 115-3 | 2008, mis en ligne le 30 septembre 2008, consulté le 22 juillet 2022. URL : http://journals.openedition.org/abpo/299 ; DOI : https://doi.org/10.4000/abpo. 299

Ce document a été généré automatiquement le 22 juillet 2022.

Tous droits réservés 


\title{
Jean-Pierre LeTHUILLIER (dir), Des habits et nous. Vêtir nos identités
}

\author{
Brigitte Maillard
}

\section{RÉFÉRENCE}

Rennes, PUR, 2007, 223 p.

\section{NOTE DE L'ÉDITEUR}

Cet ouvrage sur le site des Presses universitaires de Rennes : notice, documents et commande en ligne : http://www.pur-editions.fr/detail.php?idOuv=1405

1 Ce livre a été réalisé à l'occasion d'un colloque et d'une exposition dont il porte le titre et qui est destinée à être présentée en Bretagne, en Poitou, en Normandie et en Alsace, toutes provinces dont les costumes dits régionaux ont fourni de nombreux éléments pour cette étude et sont abondamment représentés. Il est cependant bien plus qu'un catalogue illustré. Cet ouvrage est le fruit d'une coopération entre historiens, ethnologues et conservateurs de musée.

Dans une introduction méthodologique et critique Nicole Pellegrin montre bien que si les sources écrites de l'étude sont diverses et abondantes, les données matérielles que sont les pièces de vêtement elles-mêmes sont rares et fragiles mais sont désormais presque toutes connues. Ces dernières et les représentations (gravures, tableaux) qui nous sont restées forment la base de l'étude menée par Jean-Pierre Lethuillier qui, en quatre chapitres, traite de la création, l'évolution, les usages sociaux et certaines formes d'instrumentalisation des costumes dits "régionaux"; chaque chapitre se termine par un dossier documentaire très fourni, grâce à la présentation de différents vêtements, objets, outils qui font l'objet de notices très précises. 
3 Le vêtement ne sert pas seulement à protéger les corps ; il a une signification sociale et contribue à la construction de l'image que l'individu veut donner de lui ; mais il ne faut pas oublier les vêtements conservés sont le plus souvent des habits de fête; le terme de " populaire » est un abus de langage puisque ces vêtements n'étaient pas portés par le "peuple» mais bien par les petites élites locales, surtout rurales. Les vêtements quotidiens et de travail, tout comme les hardes des plus pauvres, n'ont pas subsisté, seuls les documents figurés portent témoignage à leur sujet; cependant un vêtement de travail, plus fonctionnel et mieux adapté aux besoins des différents métiers, se développe : au XIx ${ }^{e}$ siècle, c'est la blouse bleue, peu à peu remplacée par le «bleu de travail » qui a une signification sociale et non pas régionale.

4 L'extraordinaire variété des «costumes régionaux» dans le temps et dans l'espace éclate au long de ces pages. La démonstration est clairement faite qu'il n'existe pas un costume unique et typique, «traditionnel » pour une région quelle que soit la période considérée ; au contraire l'évolution a toujours existé et a parfois été très rapide. Au $\mathrm{xx}^{\mathrm{e}}$ siècle, les " costumes régionaux » sont devenus un objet de curiosité scientifique pour les ethnologues puis un argument touristique ; la «folklorisation » a abouti à une simplification abusive et au développement de stéréotypes.

De l'économie de la rareté dans les campagnes jusqu'au milieu du xvIII ${ }^{e}$ siècle, on passe peu à peu à l'abondance pour atteindre un apogée dans la quantité et la diversité des pièces de linge et de vêtements vers le milieu du xix ${ }^{e}$ siècle. Les formes changent aussi rapidement, en partie sous l'influence des modes urbaines qui ne sont pas ignorées. Une diversité et des décalages qu'il est difficile d'expliquer caractérisent les évolutions régionales: les costumes locaux en Bretagne et en Alsace présentent une grande richesse et une longévité qui ne s'observe pas dans d'autres régions. La variété des costumes à l'intérieur d'une même province peut s'expliquer en partie par le mode de fabrication qui est local, dans de petits ateliers ou à domicile, et ignore l'uniformisation $\mathrm{du}$ " prêt à porter »; il sait adopter les nouvelles matières (le développement de l'usage du coton répond en partie aux besoins croissants de confort et d'hygiène), de nouvelles formes (qui souvent prennent leur aspect le plus spectaculaire dans les coiffes : coiffes cauchoises, coiffes du pays bigouden dont l'étirement atteint son apogée au milieu du $\mathrm{xx}^{\mathrm{e}}$ siècle), de nouvelles techniques (l'introduction des machines à broder a permis le renouvellement des motifs). L'évolution du costume local a aussi une signification sociale et économique. Il existe des codes vestimentaires qui reposent sur des usages sociaux et non des interdits réglementaires; ils permettent d'identifier sans mal les individus, selon leur âge, leur situation sociale ou matrimoniale etc., donc de déterminer le groupe auquel ils appartiennent. Les vêtements s'adaptent aussi aux nouveaux besoins, comme ceux dus au développement des loisirs. À la fin du XIx et au début $\mathrm{du} \mathrm{xx}^{\mathrm{e}}$ siècle les costumes "régionaux» ont fait l'objet d'une récupération multiforme, politique (en Alsace par exemple) puis publique et touristique (en Bretagne entre autres).

6 Ce livre est d'abord un très beau livre d'images qu'on prend plaisir à feuilleter mais il est aussi un ouvrage scientifique qui apporte des mises au point utiles sur un thème essentiel dans l'histoire de l'économie, de la culture matérielle et des faits culturels et ouvre des pistes de recherche pour dépasser la «folklorisation» des costumes « régionaux ». 